\title{
Screening of Little Millet Germplasm against Grain Smut Caused by Macalpinomyces sharmae
}

\author{
Ashwini Kumar ${ }^{1}$, A.K. Jain ${ }^{2}$, Purnima Singh ${ }^{1}$ and Narayan Lal ${ }^{3}$ \\ ${ }^{1}$ Department of Plant Pathology, JNKVV, Jabalpur, India \\ ${ }^{2}$ Department of Plant Pathology, CoA, Rewa, JNKVV, Jabalpur, India \\ ${ }^{3}$ Department of Horticulture, JNKVV, Jabalpur, India \\ *Corresponding author
}

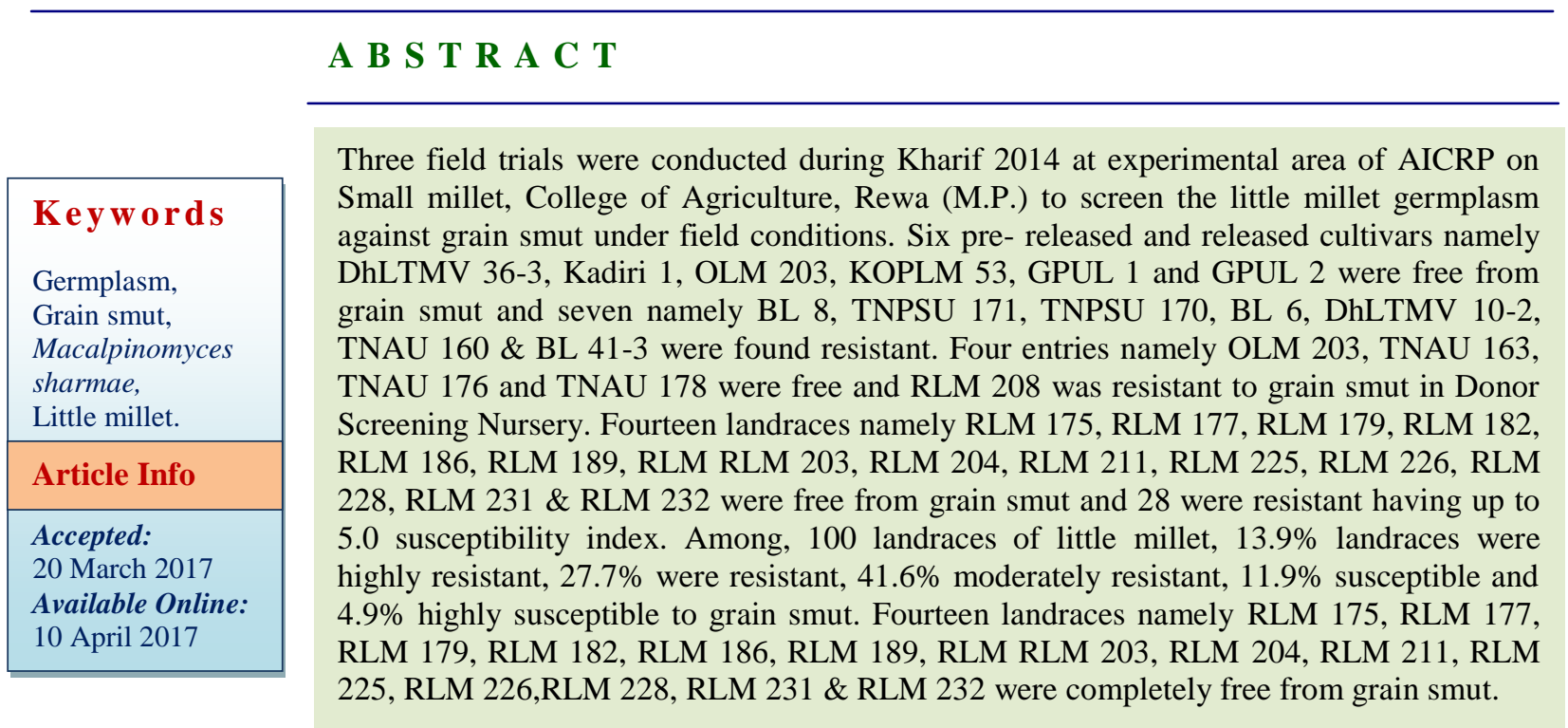

\section{Introduction}

Little millet (Panicum sumatrense Roth ex Roemer and Schultes), locally known as kutki, mejhari, medois one of the hardiest minor cereal crop belonging to the family Poaceae (Gramineae) and is indigenous to Indian sub-continent. The crop is cultivated by tribal and poor farmers in low fertile soils with low or no cash input for food and feed. It has an excellent rejuvenating capacity compared to other cereal crops. In India, the crop is cultivated in an area of 291 thousand hectares with annual production of 102 thousand tones and productivity of $349 \mathrm{~kg}$ per hectare (Anonymous, 2011) which is very less as compared to other cereal crops. Andhra Pradesh, Chhattisgarh, Madhya Pradesh, Odisa, Tamil Nadu, Karnataka, Jharkhand and Gujarat are major little millet growing states in the country. In Madhya Pradesh, the crop is cultivated in 51.54 thousand hectare with productivity of $525.5 \mathrm{~kg}$ per hectare (www.landrecords.mp.gov.in). Dindori, Mandla, Chhindwara, Balaghat, Seoni, Anuppur, Betal, Singrauli, Umaria, Sidhi, Shahdol, Jabalpur, Narsinghpur, Raisen and Khandwa are major little millet growing 
districts of Madhya Pradesh. The crop is highly drought tolerant and nutritionally as well as medicinally superior or at par with other cultivated cereals. Grains are recommended for diabetic and patients of cardio-vascular diseases. The grain of little millet possesses excellent storage properties and can be stored for several years without fear of store grain pests under ordinary storage conditions. Little millet is well known for its drought tolerance and is considered as one of the least water demanding crop. Being eco-friendly, the crop is suitable for fragile and vulnerable agro-ecosystems. Grain smut (Macalpinomyces sharmae), rust (Uromyces linearis), banded leaf and sheath blight (Rhizoctonia solani) and Udbatta (Ephelis oryzae) are important fungal diseases occurred at different stages of plant growth and caused economical yield loss under favourable environmental conditions (Pall et al., 1980; Jain et al., 1997 and Chauhan, 2014). In India, grain smut was first reported by Sharma and Khare (1987) from Dindori district of Madhya Pradesh and causal organism was identified as Tolyposporium sp. Later, it was described as Macalpinomyces sharmae (Vanky, 1995). The disease is also reported from Jharkhand, Chhattisgarh and Tamil Nadu states of the country (Anonymous, 2004 and 2012; Haider, 1997). Sharma and Khare (1987) noticed up to 50 per cent plants/ grains affected by the pathogen whereas Jain et al., (2006) reported 9.8 to 53.5 per cent reduction in grain yield per plant, 4.2 to 16.6 per cent in plant height and 6.4 to 38.9 per cent in panicle length. Jain and Joshi (2015) recorded 2.1 to $18.9 \%$ loss in grain yield due to grain smut in little millet.

The disease is ovaricolous and symptoms appeared at grain formation stage. The affected ovary is converted into smut, but does not increase in size than the normal grain. Some of the late developing grains remain greenish and increase in size slightly over the normal grains. On pressing such greenish healthy appearing grains release spores (Sharma and Khare, 1987). Studies on management of grain smut in little millet is meager in the literature, however few studies on identification of resistant sources and management through seed treatment with carboxin and carbendazim has been reported. Although, disease can be controlled by application of different chemicals but this is not the right way to control diseases in the present context because chemical application has its many disadvantages like soil pollution, water pollution and environment hazardous. Now a day people are very conscious to health and they are moving to organic production and consumption. The growing of resistant genotypes of crops is one of the best ways to manage many biotic and abiotic stresses in organic crop production system. So the present study was focused on status of the disease in the farmers' field and identification of resistant sources.

\section{Materials and Methods}

\section{Pre-released and released cultivars of little millet}

Twenty pre-released and released cultivars (DhLtMV 36-3, TNPSU 174, BL 8, Kadiri 1, DLM 89, TNPSU 167, BL 150, OLM 203, DLM 103, TNPSU 171, JK 8, TNPSU 170, BL 6, DhLtMV 10-2, TNAU 160, BL 41-3, KOPLM 53, GPUL 1, GPUL 2 and JK 36) of little millet having different maturity period were screened against grain smut. The seeds were sown in two rows of $3.0 \mathrm{~m}$ length in three replications at the spacing of $25.0 \mathrm{~cm}$ row to row and $7.5 \mathrm{~cm}$ plant to plant in randomized block design. Recommended doses of fertilizer i.e. $40 \mathrm{~kg} \mathrm{~N}$ and $20 \mathrm{~kg} \mathrm{P}_{2} \mathrm{O}_{5}$ were applied before sowing for optimum plant growth. Grain smut incidence was recorded at dough stage by counting healthy and smutted plants in each row. Grain smut 
severity was recorded by counting infected grains per panicle and susceptibility index (SI) was calculated for each cultivars.

\section{Reaction of little millet cultivars in donor screening nursery}

Thirteen cultivars (RLM 135, RLM 175, RLM 192, RLM 203, RLM 204, RLM 208, RLM 224, JK 8, OLM 203, TNAU 163, TNAU 176, TNAU 178 and RLM 4-1) of little millet received from Project Coordinating cell (Small millets), Bangalore, were sown in two rows of $3.0 \mathrm{~m}$ length in three replications at the spacing of $25.0 \mathrm{~cm}$ row to row and $7.5 \mathrm{~cm}$ plant to plant in randomized block design. Recommended doses of fertilizer i.e. $40 \mathrm{~kg} \mathrm{~N}$ and $20 \mathrm{~kg} \mathrm{P}_{2} \mathrm{O}_{5}$ were applied before sowing for optimum plant growth. Grain smut incidence was recorded at dough stage by counting healthy and smutted plants in each row. Grain smut severity was recorded by counting infected grains per panicle and susceptibility index (SI) was calculated for each cultivar.

\section{Land races of little millet}

One hundred land races collected from seven districts of Madhya Pradesh were screened along with susceptible check (JK 8) against grain smut under field condition. The Seeds were sown in two rows of $3.0 \mathrm{~m}$ length with $25.0 \mathrm{~cm}$ row to row and $7.5 \mathrm{~cm}$ plant to plant spacing in augmented design. Recommended doses of fertilizer i.e. $40 \mathrm{~kg} \mathrm{~N}$ and $20 \mathrm{~kg} \mathrm{P}_{2} \mathrm{O}_{5}$ were applied before sowing for optimum plant growth. Grain smut incidence was recorded by counting healthy and smutted plants in each row at dough stage. Grain smut severity was recorded in 20 panicles and susceptibility index was calculated.

Identification and utilization of resistant cultivars is the cheapest and feasible way to combat with any disease problem. In the present study, 20 pre-released and released cultivars, 13 entries from Donor Screening Nursery (DSN) and 100 landraces of little millet were screened against grain smut under field conditions. Six pre-released and released cultivars namely DhLTMV 36-3, Kadiri 1, OLM 203, KOPLM 53, GPUL 1 and GPUL 2 were free from grain smut and seven namely BL 8, TNPSU 171, TNPSU 170, BL 6, DhLTMV 10-2, TNAU 160 and BL 41-3 have shown resistance. In DSN, four entries namely OLM 203, TNAU 163, TNAU 176 and TNAU 178 were free from grain smut and RLM 208 was resistant. Among 100 landraces of little millet, 14 landraces namely RLM 175, RLM 177, RLM 179, RLM 182, RLM 186, RLM 189, RLM RLM 203, RLM 204, RLM 211, RLM 225, RLM 226,RLM 228, RLM 231 and RLM 232 were free from grain smut whereas 28 were resistant showing up to 5.0 susceptibility index. These cultivars may be utilized for resistance breeding programme. Earlier few studies, for identification of resistant sources against grain smut of little millet were undertaken by Jain (2002), Jain (2003). Jain et al., (2006) and Jain and Tripathi (2007) and little millet variety OLM 203 was reported resistant while JK 8 susceptible to grain smut. These reports are in agreement with the present findings.

\section{Results and Discussion}

\section{Screening of little millet cultivars against grain smut}

Different categories of little millet cultivars i.e. pre- release and released cultivars, Donor Screening Nursery and land races exhibited significant variation in their response to grain smut caused by Macalpinomyces sharmae under field conditions. Grain smut incidence, severity and susceptibility index in 20 prereleased and released cultivars of little millet are presented in table 1. Grain smut incidence ranged 0.0 to $56.8 \%$ with 0.0 to $6.7 \%$ smut 
severity. Susceptibility index (SI) varied from 0.0 to $17.2 \%$ was maximum in $\mathrm{JK} 36$ followed by DLM $89(15.6 \%)$ and JK 8 (14.4\%). Six cultivars namely DhLTMV 363, Kadiri 1, OLM 203, KOPLM 53, GPUL 1 and GPUL 2 were free from grain smut and showed highly resistant reaction, whereas seven germplasm namely BL 8, TNPSU 171, TNPSU 170, BL 6, DhLTMV 10-2, TNAU 160 and BL 41-3 had shown resistant reaction. Three cultivars namely TNPSU 167 , BL 150 and DLM 103 were moderately resistant, two germplasm namely TNPSU 174 and JK 8 were susceptible and two germplasm namely DLM 89 and JK 36 was highly susceptible to grain smut.

Thirteen germplasm of little millet including one resistant check OLM 203 and one susceptible check JK 8 were screened against grain smut. The data presented in table 2 and variation in grain smut parameters indicated the significant differences among genotypes for their reaction to grain smut.

Table.1 Reaction of pre-released and released little millet cultivars against grain smut

\begin{tabular}{|l|l|c|c|c|c|}
\hline \multirow{2}{*}{ S. No. } & \multirow{2}{*}{ Germplasm } & \multicolumn{3}{|c|}{ Grain smut } & \multirow{2}{*}{ Reaction } \\
\cline { 3 - 5 } & & & Incidence $(\%)^{\mathrm{a}}$ & Severity $(\%)^{\mathrm{b}}$ & $\begin{array}{l}\text { Susceptibility } \\
\text { Index (SI) }\end{array}$ \\
\hline 1. & DhLtMV 36-3 & $0.0(0.00)$ & $0.0(0.701)$ & 0.0 & HR \\
\hline 2. & TNPSU 174 & $55.7(48.33)$ & $2.2(1.654)$ & 11.1 & S \\
\hline 3. & BL 8 & $30.4(33.44)$ & $0.7(1.077)$ & 4.6 & $\mathrm{R}$ \\
\hline 4. & Kadiri 1 & $0.0(0.00)$ & $0.0(0.701)$ & 0.0 & HR \\
\hline 5. & DLM 89 & $56.8(48.97)$ & $4.3(2.189)$ & 15.6 & HS \\
\hline 6. & TNPSU 167 & $34.5(35.91)$ & $1.4(1.382)$ & 6.9 & MR \\
\hline 7. & BL 150 & $27.1(31.11)$ & $1.2(1.302)$ & 5.7 & MR \\
\hline 8. & OLM 203* & $0.0(0.00)$ & $0.0(0.701)$ & 0.0 & HR \\
\hline 9. & DLM 103 & $35.7(36.57)$ & $2.1(1.604)$ & 8.7 & MR \\
\hline 10. & TNPSU 171 & $19.5(26.13)$ & $0.8(1.119)$ & 3.9 & R \\
\hline 11. & JK 8** & $42.6(40.68)$ & $4.9(2.327)$ & 14.4 & S \\
\hline 12. & TNPSU 170 & $24.1(29.27)$ & $0.7(1.077)$ & 4.1 & R \\
\hline 13. & BL 6 & $22.1(27.72)$ & $0.4(0.924)$ & 3.0 & R \\
\hline 14. & DhLtMV 10-2 & $21.1(26.93)$ & $0.6(1.028)$ & 3.6 & R \\
\hline 15. & TNAU 160 & $10.1(18.39)$ & $0.6(1.037)$ & 2.5 & R \\
\hline 16. & BL 41-3 & $13.7(21.26)$ & $0.5(1.005)$ & 2.6 & R \\
\hline 17. & KOPLM 53 & $0.0(0.00)$ & $0.0(0.701)$ & 0.0 & HR \\
\hline 18. & GPUL 1 & $0.0(0.00)$ & $0.0(0.701)$ & 0.0 & HR \\
\hline 19. & GPUL 2 & $0.0(0.00)$ & $0.0(0.701)$ & 0.0 & HR \\
\hline 20. & JK 36 & $44.4(41.75)$ & $6.7(2.673)$ & 17.2 & HS \\
\hline & CD $(0.05)$ & 7.603 & 0.248 & - & - \\
\hline
\end{tabular}

${ }^{a}$ Figures in parentheses are ARC SIN transformed values.

${ }^{\mathrm{b}}$ Figures in parentheses are square root transformed value.

*Resistant check ** Susceptible check,

$\mathrm{HR}=$ Highly Resistant,$\quad \mathrm{R}=$ Resistant,$\quad \mathrm{MR}=$ Moderately Resistant,

$\mathrm{S}=$ Susceptible, $\mathrm{HS}=$ Highly Susceptible 
Table.2 Reaction of little millet entries against grain smut in donor screening nursery

\begin{tabular}{|l|l|c|c|c|c|}
\hline $\begin{array}{l}\text { S. } \\
\text { No. }\end{array}$ & Germplasm & $\begin{array}{l}\text { Grain } \\
\text { Incidence (\%) }^{\text {smut }}\end{array}$ & $\begin{array}{c}\text { Grain } \\
\text { severity (\%) }\end{array}$ & $\begin{array}{c}\text { Smut }^{\mathbf{6}} \\
\text { index (SI) }\end{array}$ & Reaction \\
\hline 1. & RLM 135 & $35.6(36.60)$ & $2.7(1.776)$ & 9.8 & MR \\
\hline 2. & RLM 175 & $41.4((39.93)$ & $3.1(1.860)$ & 11.3 & S \\
\hline 3. & RLM 192 & $41.3(39.89)$ & $1.7(1.477)$ & 8.4 & MR \\
\hline 4. & RLM 203 & $57.1(49.15)$ & $2.7(1.774)$ & 12.4 & S \\
\hline 5. & RLM 204 & $61.0(51.43)$ & $3.3(1.942)$ & 14.2 & S \\
\hline 6. & RLM 208 & $25.5(30.16)$ & $0.8(1.111)$ & 4.5 & R \\
\hline 7. & RLM 224 & $47.2(43.34)$ & $2.8(1.804)$ & 11.5 & S \\
\hline 8. & JK 8** & $69.4(57.00)$ & $5.4(2.436)$ & 19.4 & HS \\
\hline 9. & OLM 203* & $0.0(0.00)$ & $0.0(0.701)$ & 0.0 & HR \\
\hline 10. & TNAU 163 & $0.0(0.00)$ & $0.0(0.701)$ & 0.0 & HR \\
\hline 11. & TNAU 176 & $0.0(0.00)$ & $0.0(0.701)$ & 0.0 & HR \\
\hline 12. & TNAU 178 & $0.0(0.00)$ & $0.0(0.701)$ & 0.0 & HR \\
\hline 13. & RLM 4-1 & $28.4(32.13)$ & $0.9(0.701)$ & 5.1 & MR \\
\hline & CD(0.05) & 9.424 & 0.367 & - & - \\
\hline
\end{tabular}

${ }^{a}$ Figures in parentheses are ARC SIN transformed values

${ }^{\mathrm{b}}$ Figures in parentheses are square root transformed value.

*Resistant check, ** Susceptible check

HR = Highly Resistant,$\quad \mathrm{R}=$ Resistant,$\quad \mathrm{MR}=$ Moderately Resistant,

$\mathrm{S}=$, Susceptible, HS= Highly Susceptible

Table.3 Reaction of little millet land races against grain smut under field conditions

\begin{tabular}{|l|l|c|c|c|c|}
\hline \multirow{2}{*}{ S No. } & \multirow{2}{*}{ Germplasm } & \multicolumn{3}{|c|}{ Grain smut } & \multirow{2}{*}{ Reaction } \\
\cline { 3 - 5 } & $\begin{array}{c}\text { Grain smut } \\
\text { Incidence } \\
(\%)\end{array}$ & $\begin{array}{c}\text { Grain smut } \\
\text { Severity } \\
(\%)\end{array}$ & $\begin{array}{c}\text { Susceptibility } \\
\text { index (SI) }\end{array}$ & \\
\hline 1. & RLM 101 & 23.0 & 2.5 & 7.6 & MR \\
\hline 2. & RLM 102 & 26.7 & 6 & 12.7 & S \\
\hline 3. & RLM 103 & 20.6 & 1.8 & 6.1 & MR \\
\hline 4. & RLM 104 & 7.7 & 2.3 & 4.2 & R \\
\hline 5. & RLM 105 & 66.7 & 6.1 & 20.2 & HS \\
\hline 6. & RLM 106 & 14.3 & 2.3 & 5.7 & MR \\
\hline 7. & RLM 108 & 14.3 & 1.4 & 4.5 & R \\
\hline 8. & RLM 109 & 30.0 & 1.4 & 6.5 & MR \\
\hline 9. & RLM 110 & 64.3 & 3.5 & 15.0 & S \\
\hline 10. & RLM 112 & 33.3 & 2.2 & 8.6 & MR \\
\hline 11. & RLM 113 & 35.7 & 5.1 & 13.5 & S \\
\hline 12. & RLM 114 & 10.0 & 8.7 & 9.3 & MR \\
\hline 13. & RLM 115 & 23.1 & 1.7 & 6.3 & MR \\
\hline 14. & RLM 116 & 16.7 & 3.0 & 7.1 & MR \\
\hline & & & & & \\
\hline
\end{tabular}




\begin{tabular}{|c|l|c|c|c|c|}
\hline S No. & Germplasm & \multicolumn{3}{|c|}{ Grain smut } & \multirow{2}{*}{ Reaction } \\
\cline { 3 - 5 } & & $\begin{array}{c}\text { Grain smut } \\
\text { Incidence } \\
(\%)\end{array}$ & $\begin{array}{c}\text { Grain smut } \\
\text { Severity } \\
(\%)\end{array}$ & $\begin{array}{c}\text { Susceptibility } \\
\text { index (SI) }\end{array}$ & \\
\hline 15. & RLM 117 & 15.0 & 1.3 & 4.4 & R \\
\hline 16. & RLM 119 & 13.3 & 2.1 & 5.3 & MR \\
\hline 17. & RLM 120 & 19.4 & 1.6 & 5.6 & MR \\
\hline 18. & RLM 121 & 10.0 & 0.7 & 2.6 & R \\
\hline 19. & RLM 122 & 11.8 & 2.1 & 5.0 & R \\
\hline 20. & RLM 126 & 13.3 & 1.5 & 4.5 & R \\
\hline 21. & RLM 127 & 27.8 & 8.2 & 15.1 & HS \\
\hline 22. & RLM 128 & 20.0 & 1.3 & 5.1 & MR \\
\hline 23. & RLM 129 & 21.1 & 1.3 & 5.2 & MR \\
\hline 24. & RLM 130 & 7.1 & 1.9 & 3.7 & R \\
\hline 25. & RLM 134 & 16.7 & 2.1 & 5.9 & MR \\
\hline 26. & RLM 135 & 21.4 & 3.4 & 8.5 & MR \\
\hline 27. & RLM 138 & 21.7 & 1.3 & 5.3 & MR \\
\hline 28. & RLM 139 & 20.0 & 3.1 & 7.9 & MR \\
\hline 29. & RLM 140 & 36.4 & 3.0 & 10.4 & S \\
\hline 30. & RLM 141 & 23.1 & 3.5 & 9.0 & MR \\
\hline 31. & RLM 142 & 23.5 & 3.0 & 8.4 & MR \\
\hline 32. & RLM 143 & 45.4 & 7.9 & 18.9 & HS \\
\hline 33. & RLM 144 & 19.4 & 1.6 & 5.6 & MR \\
\hline 34. & RLM 146 & 32.8 & 2.6 & 9.2 & MR \\
\hline 35. & RLM 147 & 17.6 & 1.9 & 5.8 & MR \\
\hline 36. & RLM 148 & 25.0 & 3.5 & 9.4 & MR \\
\hline 37. & RLM 149 & 53.3 & 7.5 & 20.0 & HS \\
\hline 38. & RLM 150 & 17.6 & 2.3 & 6.4 & MR \\
\hline 39. & RLM 151 & 13.3 & 1.4 & 4.3 & R \\
\hline 40. & RLM 152 & 16.7 & 1.3 & 4.7 & R \\
\hline 41. & RLM 153 & 21.4 & 3.6 & 8.8 & MR \\
\hline 42. & RLM 154 & 14.9 & 1.4 & 4.6 & R \\
\hline 43. & RLM 155 & 41.7 & 2.8 & 10.8 & S \\
\hline 44. & RLM 156 & 25.0 & 3.0 & 8.7 & MR \\
\hline 45. & RLM 157 & 19.3 & 1.7 & 5.7 & MR \\
\hline 46. & RLM 158 & 38.5 & 4.1 & 12.6 & S \\
\hline 47. & RLM 160 & 17.6 & 3.6 & 8.0 & MR \\
\hline 48 & RLM 161 & 7.7 & 1.7 & 3.6 & R \\
\hline 49 & RLM 162 & 21.1 & 0.4 & 2.9 & R \\
\hline 50 & RLM 165 & 20.0 & 1.2 & 4.9 & R \\
\hline 51 & RLM 166 & 7.7 & 1.4 & 3.3 & R \\
\hline 52 & RLM 167 & 30.8 & 2.5 & 8.8 & MR \\
\hline 53 & RLM 170 & 15.4 & 1.3 & 4.5 & R \\
\hline 54 & RLM 171 & 7.8 & 1.1 & 2.9 & R \\
\hline
\end{tabular}




\begin{tabular}{|l|l|c|c|c|c|}
\hline S No. & Germplasm & \multicolumn{3}{|c|}{ Grain smut } & \multirow{2}{*}{ Reaction } \\
\cline { 3 - 6 } & & $\begin{array}{c}\text { Grain smut } \\
\text { Incidence } \\
(\%)\end{array}$ & $\begin{array}{c}\text { Grain smut } \\
\text { Severity } \\
(\%)\end{array}$ & $\begin{array}{c}\text { Susceptibility } \\
\text { index (SI) }\end{array}$ & \\
\hline 55 & RLM 172 & 22.2 & 1.2 & 5.2 & MR \\
\hline 56 & RLM 173 & 7.7 & 3.3 & 5.0 & R \\
\hline 57 & RLM 174 & 33.3 & 2.6 & 9.3 & MR \\
\hline 58 & RLM 175 & 0.0 & 0.0 & 0.0 & HR \\
\hline 59 & RLM 176 & 25.0 & 1.7 & 6.5 & MR \\
\hline 60 & RLM 177 & 0.0 & 0.0 & 0.0 & HR \\
\hline 61 & RLM 178 & 25.0 & 3.5 & 9.4 & MR \\
\hline 62 & RLM 179 & 0.0 & 0.0 & 0.0 & HR \\
\hline 63 & RLM 181 & 20.0 & 2.9 & 7.6 & MR \\
\hline 64 & RLM 182 & 0.0 & 0.0 & 0.0 & HR \\
\hline 65 & RLM 183 & 23.1 & 1.1 & 5.0 & R \\
\hline 66 & RLM 185 & 41.1 & 2.5 & 10.1 & S \\
\hline 67 & RLM 186 & 0.0 & 0.0 & 0.0 & HR \\
\hline 68 & RLM 189 & 0.0 & 0.0 & 0.0 & HR \\
\hline 69 & RLM 191 & 6.3 & 1.3 & 2.9 & R \\
\hline 70 & RLM 193 & 23.1 & 2.5 & 7.6 & MR \\
\hline 71 & RLM 194 & 16.7 & 3.0 & 7.1 & MR \\
\hline 72 & RLM 195 & 8.3 & 1.4 & 3.4 & R \\
\hline 73 & RLM 196 & 53.3 & 3.2 & 13.1 & S \\
\hline 74 & RLM 198 & 18.0 & 1.3 & 4.8 & R \\
\hline 75 & RLM 199 & 50.0 & 3.2 & 12.6 & S \\
\hline 76 & RLM 200 & 25.0 & 1.8 & 6.7 & MR \\
\hline 77 & RLM 201 & 19.2 & 1.2 & 4.8 & R \\
\hline 78 & RLM 202 & 6.2 & 1.3 & 2.8 & R \\
\hline 79 & RLM 203 & 0.0 & 0.0 & 0.0 & HR \\
\hline 80 & RLM 204 & 0.0 & 0.0 & 0.0 & HR \\
\hline 81 & RLM 205 & 11.7 & 1.3 & 3.9 & R \\
\hline 82 & RLM 206 & 13.3 & 2.8 & 6.1 & MR \\
\hline 83 & RLM 207 & 7.1 & 2.1 & 3.9 & R \\
\hline 84 & RLM 211 & 0.0 & 0.0 & 0.0 & HR \\
\hline 85 & RLM 215 & 42.2 & 2.2 & 9.6 & MR \\
\hline 86 & RLM 216 & 15.4 & 2.1 & 5.7 & MR \\
\hline 87 & RLM 217 & 12.1 & 2.3 & 5.3 & MR \\
\hline 88 & RLM 218 & 22.2 & 2.7 & 7.7 & MR \\
\hline 89 & RLM 219 & 29.0 & 2.8 & 9.0 & MR \\
\hline 90 & RLM 220 & 41.7 & 4.6 & 13.8 & S \\
\hline 91 & RLM 222 & 46.1 & 7.1 & 18.1 & HS \\
\hline 92 & RLM 223 & 16.3 & 1.3 & 4.6 & R \\
\hline 93 & RLM 224 & 41.7 & 2.7 & 10.6 & S \\
\hline 94 & RLM 225 & 0.0 & 0.0 & 0.0 & HR \\
\hline & & & & & \\
\hline & & & & & \\
\hline
\end{tabular}




\begin{tabular}{|l|l|c|c|c|c|}
\hline \multirow{2}{*}{ S No. } & \multirow{2}{*}{ Germplasm } & \multicolumn{3}{|c|}{ Grain smut } & \multirow{2}{*}{ Reaction } \\
\cline { 3 - 6 } & $\begin{array}{c}\text { Grain smut } \\
\text { Incidence } \\
(\%)\end{array}$ & $\begin{array}{c}\text { Grain smut } \\
\text { Severity } \\
(\%)\end{array}$ & $\begin{array}{c}\text { Susceptibility } \\
\text { index (SI) }\end{array}$ & \\
\hline 95 & RLM 226 & 0.0 & 0.0 & 0.0 & HR \\
\hline 96 & RLM 227 & 15.1 & 1.3 & 4.4 & R \\
\hline 97 & RLM 228 & 0.0 & 0.0 & 0.0 & HR \\
\hline 98 & RLM 229 & 6.2 & 2.9 & 4.2 & R \\
\hline 99 & RLM 231 & 0.0 & 0.0 & 0.0 & HR \\
\hline 100 & RLM 232 & 0.0 & 0.0 & 0.0 & HR \\
\hline 101 & JK 8 (SC) & 43.1 & 5.1 & 14.8 & S \\
\hline & Mean & 20.0 & 2.3 & 6.5 & \\
& Max. & 66.7 & 8.7 & 20.2 & \\
& Min & 0.0 & 0.0 & 0.0 & \\
& SD & 14.73 & 1.83 & 4.56 & \\
\hline
\end{tabular}

Table.4 Grouping of little millet land races on the basis of grain smut susceptibility index (SI)

\begin{tabular}{|c|c|c|c|}
\hline Reaction & $\begin{array}{c}\text { Grain } \\
\text { smut }(\mathrm{SI})\end{array}$ & $\begin{array}{c}\text { No. of } \\
\text { landraces }\end{array}$ & germplasm \\
\hline HR & 0.0 & $\begin{array}{l}14 \\
(13.9 \%)\end{array}$ & $\begin{array}{l}\text { RLM } 175,177,179,182,186,189,203,204, \\
211,225,226,228,231 \text { and } 232\end{array}$ \\
\hline $\mathrm{R}$ & Up to 5 & $\begin{array}{c}28 \\
(27.7 \%)\end{array}$ & $\begin{array}{l}\text { RLM } 104,108,117,121,122,126,130,151,152, \\
154,161,162,165,166, \quad 170,171,173,183,191, \\
195,198,201,202,205,207,223,227,229\end{array}$ \\
\hline MR & 5.1 to 10.0 & $\begin{array}{c}42 \\
(41.6 \%)\end{array}$ & $\begin{array}{l}\text { RLM } 101,103,106,109,112,114,115,116, \\
119,120,128,129,131,135,138,139,141, \\
142,144,146,147,148,150,153,156,157, \\
160,167,172,174,176,178,181,193,194, \\
200,206,215,216,217,218 \text { and } 219\end{array}$ \\
\hline $\mathrm{S}$ & 10.0 to 15 & $\begin{array}{l}12 \\
(11.9 \%)\end{array}$ & $\begin{array}{l}\text { RLM 102, 110, 113, 140, 155, 158, 185, 196, } \\
\text { 199, 220, 224, JK } 8\end{array}$ \\
\hline HS & $<15$ & $\begin{array}{c}5 \\
(4.9 \%)\end{array}$ & RLM $105,127,143,149,222$ \\
\hline
\end{tabular}

Figures in parentheses are percentage of land races.

$\mathrm{HR}=$ Highly resistant, $\mathrm{R}=$ Resistant, MR= Moderately Resistant,

$\mathrm{S}=$ Susceptible, HS= Highly Susceptible,

The disease incidence, severity and susceptibility index varied from 0.0 to $69.4 \%$, 0.0 to $5.4 \%$ and 0.0 to $19.4 \%$, respectively. Four germplasm namely OLM 203, TNAU 163, TNAU 176 and TNAU 178 found highly resistant showing $0.0 \%$ grain smut incidence, whereas RLM 208 was found resistant to grain smut. Three germplasm namely RLM 135, RLM 192 and RLM 4-1 were moderately resistant, four namely RLM 175, RLM 203, RLM 204 and RLM 224 were susceptible and JK 8 was highly susceptible to grain smut. 
Incidence of grain smut in 101 land races of little millet including one susceptible check JK 8 is presented in table 3. Significant variation among land races for their reaction to grain smut was observed. Grain smut incidence varied from 0.0 to $66.7 \%$ with a mean of $20 \%$ among the screened land races. Smut severity ranging from 0.0 to $8.7 \%$ with a mean of $2.3 \%$ and susceptibility index (SI) varied from 0.0 to $20.2 \%$ with a mean of 6.5 . Fourteen land races namely RLM 175, RLM 177, RLM 179, RLM 182, RLM 186, RLM 189, RLM 203, RLM 204, RLM 211, RLM 225, RLM 226,RLM 228, RLM 231 and RLM 232 were free from grain smut, whereas 28 land races namely RLM 104, RLM 108, RLM 117， RLM 121， RLM 122,RLM 126,RLM 130,RLM 151,RLM 152, RLM 154, RLM 161, RLM 162, RLM 165, RLM 166, RLM 170, RLM 171, RLM 173, RLM 183, RLM 191, RLM 195, RLM 198, RLM 201, RLM 202, RLM 205, RLM 207, RLM 223, RLM 227 and RLM 229 were resistant showing up to 5.0 susceptibility index. Forty two land races were with moderately resistant reaction of which 12 namely RLM 102, RLM 110, RLM 113, RLM 140, RLM 155, RLM 158, RLM 185, RLM 196, RLM 199, RLM 220, RLM 224 and JK 8 were susceptible and 5 namely RLM 105, RLM 127, RLM 143, RLM 149 and RLM 222 were highly susceptible to grain smut. Data presented in table 4 revealed that $41.7 \%$ land races were MR, $27.7 \%$ were $\mathrm{R}$ and $13.9 \%$ were highly resistant to grain smut. While only $11.9 \%$ land races were susceptible and $4.9 \%$ were highly susceptible to grain smut.

Identification and utilization of resistant cultivars is the cheapest and feasible way to combat with any disease problem. Six prereleased and released cultivars namely DhLTMV 36-3, Kadiri 1, OLM 203, KOPLM 53, GPUL 1 and GPUL 2 were free from grain smut and seven namely BL 8, TNPSU 171, TNPSU 170, BL 6, DhLTMV 10-2,
TNAU 160 and BL 41-3 have shown resistance. In DSN, four entries namely OLM 203, TNAU 163, TNAU 176 and TNAU 178 were free from grain smut and RLM 208 was resistant. Among 100 landraces of little millet, 14 landraces namely RLM 175, RLM 177, RLM 179, RLM 182, RLM 186, RLM 189, RLM RLM 203, RLM 204, RLM 211, RLM 225, RLM 226, RLM 228, RLM 231 and RLM 232 were free from grain smut whereas 28 were resistant showing up to 5.0 susceptibility index. These germplasm may be utilized for resistance breeding programme. Earlier few studies, for identification of resistant sources against grain smut of little millet were undertaken by Jain (2002), Jain (2003). Jain et al., (2006) and Jain and Tripathi (2007) and little millet variety OLM 203 was reported resistant while JK 8 susceptible to grain smut.

\section{Acknowledgement}

The authors are thankful to Project Coordinator, AICRP on Small millet, College of Agriculture, Rewa (M.P.), Staff of Department of Plant Pathology, College of Agriculture, Rewa and my seniors, for providing critical suggestion and appropriate guidance's to carry out the experiment successfully during the period of research work.

\section{References}

Anonymous. 2004. Annual Report (2003-04) of All India Coordinated Small Millets Improvement Project. ICAR, GKVK, Bangalore, India. pp- 51.

Anonymous. 2011. Coordinators Review paper presented in annual workshop of AICRP on small millets, held at OUAT, Bhubaneshwar (Odisha) on April 23-25.

Anonymous. 2012. Annual Report (2011-12) of All India Coordinated Small Millets Improvement Project. ICAR, GKVK, 
Bangalore, India, pp- 44.

Chauhan, S.S. 2014. Studies on banded leaf and sheath blight of little millet (Panicum sumatrense) caused by Rhizoctonia solani Kuhn. M.Sc. (Agri.) Thesis, J.N.K.V.V. Jabalpur (MP). India pp. 1-95.

Haider, Z.A. 1997. Little millet in Indian Agriculture: Progress and Perspectives. In Nat. Seminar on Small Millets: Current trends and future priorities as food, feed and in processing for value addition held at TNAU, Coimbatore, April 23-24, 1997.

Jain, A.K. and Tripathi, S.K. 2007. Management of grain smut (Macalpinomyces sharmae) in little millet. Indian Phytopathol., 60(4): 467471.

Jain, A.K., Tripathi, S.K. and Singh, R.P. 2006. Macalpinomyces sharmae: A new threat for the cultivation of little millet in Madhya Pradesh. Proc. Nat.Symp. on "Emerging Plant diseases, their diagnosis and management", 31 to Feb,
2, 2006 at N.B.U. Siliguri (W.B.), India. pp 31-32.

Jain A.K., Yadava, H.S. and Jain, S.K. 1997. Genetic resistance against microbes in small millets. Adv. Plant Sci., 9(2) suppl:133-43.

Jain, A.K. 2002. Host plant resistance and impact of planting time on incidence of grain smut in little millet. J. Mycol. PIant Pathol., 32: 309.

Jain, A.K. 2003. Occurrence of grain smut of little millet caused by Macalpinomyces sharmae in Madhya Pradesh. PIant Protection Bull., 55: 30-32.

Pall, B.S., Jain, A.C. and Singh, S.P.1980. Diseases of lesser millets, J.N.K.V.V., Jabalpur (M.P.), pp62-69.

Sharma, N.D. and Khare, M.N. 1987. Two new smut diseases of little millet (Panicum sumatrense) from India. Acta Botanica Indica, 15: 143-144.

Vanky, K. 1995. Taxonomical studies on Ustilaginales xII. Mycotaxon., 54: 215138.

\section{How to cite this article:}

Ashwini Kumar, A.K. Jain, Purnima Singh and Narayan Lal. 2017. Screening of Little Millet Germplasm against Grain Smut Caused By Macalpinomyces sharmae. Int.J.Curr.Microbiol.App.Sci. 6(4): 2187-2196. doi: https://doi.org/10.20546/ijcmas.2017.604.256 